\title{
Iterative Learning Control for Remote Control Systems with Communication Delay and Data Dropout
}

\author{
Chunping Liu, ${ }^{1,2}$ Jianxin $X u^{3}{ }^{3}$ and Jun $W^{1}$ \\ ${ }^{1}$ State Key Laboratory of Industrial Control Technology, Institute of Cyber-Systems and Control, \\ Zhejiang University, Hangzhou 310027, China \\ ${ }^{2}$ Department of Electrical and Information Engineering, Shaoxing College of Arts and Sciences, \\ Shaoxing 31200, China \\ ${ }^{3}$ Department of Electrical and Computer Engineering, National University of Singapore, Singapore 117576
}

Correspondence should be addressed to Chunping Liu, okman_liu@hotmail.com and Jun Wu, jwu@iipc.zju.edu.cn

Received 21 October 2011; Accepted 10 January 2012

Academic Editor: Yun-Gang Liu

Copyright $@ 2012$ Chunping Liu et al. This is an open access article distributed under the Creative Commons Attribution License, which permits unrestricted use, distribution, and reproduction in any medium, provided the original work is properly cited.

Iterative learning control (ILC) is applied to remote control systems in which communication channels from the plant to the controller are subject to random data dropout and communication delay. Through analysis, it is shown that ILC can achieve asymptotical convergence along the iteration axis, as far as the probabilities of the data dropout and communication delay are known a priori. Owing to the essence of feedforward-based control ILC can perform trajectory-tracking tasks while both the data-dropout and the one-step delay phenomena are taken into consideration. Theoretical analysis and simulations validate the effectiveness of the ILC algorithm for networkbased control tasks.

\section{Introduction}

Iterative leaning control (ILC) is a control method that achieves perfect trajectory tracking when the system operates repeatedly. ILC has made significant progresses over the past two decades [1-3] and covered a wide scope of research issues such as continuous-time nonlinear system control [4], discrete-time nonlinear system [5], the initial reset problem $[6,7]$, stochastic process control [8], state delays [9], and data dropout [10].

On the other hand, the research on networked control systems has attracted much attention [11, 12] over the past few years. In network control, two frequently encountered issues are data dropout and communication delays, which are causes of poor performance of remote control systems. A central research area in remote control systems is to evaluate 
and compensate data dropout and time-delay factors [13-16]. Since data dropout and delay are random and time varying by nature, the existing control methods for deterministic data dropout and communication delays cannot be directly applied. Significant research efforts have been made on the control problems for networked systems with random data dropout and communication delays that are modeled in various ways in terms of the probability and characteristics of sources and destinations, for instance [10,17].

It is in general still an open research area in ILC when remote control systems problems are concerned, except for certain pioneer works that address linear systems associated with either random data dropout [10, 18] or random communication delays [17, 19-21]. This paper investigates the implementation of ILC in a remote control systems setting, specifically focusing on compensation when both random data dropout and delays occur at the communication channels between the plant output and the controller.

Since ILC is in principle a feedforward technique, it is possible to send the controller signal before the task is executed. This would not be possible for feedback-based control systems. Hence, the data dropout can be circumvented to certain extent by using network protocols that assure the delivery of data packets. Likewise, the large delay due to large data package can also be avoided when the package is used for repeated task executions, namely, in future executions. ILC task is carried out in a finite-time interval, hence the time-domain stability is not a concern. Thus, unlike most network control works that focus on the stability issue, ILC can be applied to address trajectory-tracking tasks and the learning convergence is achieved in the iteration domain.

On the other hand, the use of data in the feedforward fashion would require the temporal analysis and management of data packages as well as resending the missing data package, which may not be available in certain remote control systems tasks. In this work, we adopt an ILC scheme that uses pastcontrol signals, as well as the error signals that are perturbed by the data dropout and communication delay. The ILC law adopts classical Dtype algorithm and a revised learning gain that takes into consideration the probabilities of both data-dropout and communication-delay factors. As a result, the output tracking errors can be made to converge along the iteration axis. The ILC scheme can be applied to linear discrete-time plants with trajectory-tracking tasks.

The paper is organized as below. Section 2 formulates the remote control systems problem. Sections 3 and 4 prove the convergence property of ILC for linear discrete-time plants. Section 5 presents a numerical examples.

Throughout the paper, the following notations are used. Let $\varepsilon[\cdot]$ be the expected value of a random variable, $P[\cdot]$ the probability of an event, $\|\cdot\|_{2}$ the Euclidean norm of a vector, and $\|\cdot\|$ the maximal singular value of a matrix. Let $\mathbf{z}(t)$ is a discrete time signal with $t \in$ $\{0,1, \ldots, T\}$. For any $a>1$ and any $\lambda>1$, define

$$
\|\mathbf{z}\|_{(\lambda, a)} \triangleq \sup _{t \in[0, T]} a^{-\lambda t}\|\mathbf{z}(t)\|_{2}
$$

where $[0, T]=\{0,1, \ldots, T\}$.

\section{Problem Formulation}

Consider a deterministic discrete-time linear time-invariant dynamics system:

$$
\begin{gathered}
\mathbf{x}_{i}(t+1)=A \mathbf{x}_{i}(t)+B \mathbf{u}_{i}(t), \\
\mathbf{y}_{i}(t)=C \mathbf{x}_{i}(t),
\end{gathered}
$$




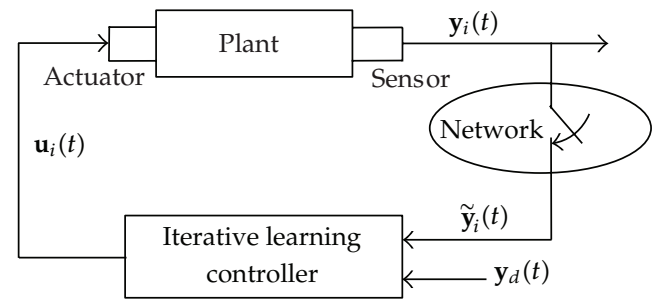

Figure 1: The schematic diagram of the remote control system.

where " $i$ " and " $t$ " denote the iteration index and discrete time, respectively. $\mathbf{x}_{i}(t) \in \mathbb{R}^{n}, \mathbf{u}_{i}(t) \in$ $\mathbb{R}^{p}$, and $\mathbf{y}_{i}(t) \in \mathbb{R}^{r}$ for all $t \in[0, T]$ are system states, inputs, and outputs, respectively, at the $i$ th iteration. $A, B$, and $C$ are constant matrices with appropriate dimensions.

The schematic diagram of the remote control systems under consideration is shown in Figure 1.

It should be noted that the open-loop system from the ILC input to the plant output is deterministic. The randomness occurs during the data transmission from the plant output to the ILC input. There are two approaches in analyzing the closed-loop system. The first approach is to treat the entire closed-loop system as a random or stochastic process. In such circumstances, the topology of the overall system keeps changing and the control process is either a Markovian jump process or a switching process. Another approach, which is adopted in this work, is to retain the essentially deterministic structure of the original openloop system, meanwhile model the random data dropout and communication delay into two random factors with known probability distributions. As a consequence, the signals used in ILC, $\tilde{\mathbf{y}}_{i}(t)$ are the modulated plant output with the two random factors. (2.1) is

When the control process is deterministic, an effective ILC law for the linear system

$$
\mathbf{u}_{i+1}(t)=\mathbf{u}_{i}(t)+L \mathbf{e}_{i}(t+1),
$$

where $\mathbf{u}_{i+1}(t)$ and $\mathbf{u}_{i}(t)$ are control inputs at the $(i+1)$ th and $i$ th iterations, namely, the present trial and the previous trial, respectively. $\mathbf{e}_{i}(t+1)=\mathbf{y}_{d}(t+1)-\mathbf{y}_{i}(t+1)$ is the output tracking error at the time $(t+1)$ th time instance of the $i$ th iteration. $L$ is a learning gain matrix.

Remark 2.1. Note that in the ILC law (2.2), the control signal of the present iteration, $\mathbf{u}_{i+1}(t)$, consists of both the pastcontrol input, $\mathbf{u}_{i}(t)$ and the past error with one-step temporal advance, $\mathbf{e}_{i}(t+1)$. The current-cycle feedback errors, such as $\mathbf{e}_{i+1}(t)$, are not used. Since ILC does not require the current-cycle feedback nor the temporal stability, it is an effective control method for remote control systems problems with random data dropout and communication delay.

To facilitate the ILC design and convergence analysis, data dropout and one-step communication delay are formulated. First formulate the data-dropout problem. Denote $r(t)$ a stochastic variable with Bernoulli distribution taking binary values 0 and 1 , where $\gamma(t)=0$ denotes an occurrence of data dropout and $\gamma(t)=1$ denotes a normal data communication. The probabilities of $\gamma(t)$ are

$$
\begin{gathered}
P[\gamma(t)=1]=\varepsilon[\gamma(t)]=\bar{\gamma}, \\
P[\gamma(t)=0]=1-\varepsilon[\gamma(t)]=1-\bar{\gamma},
\end{gathered}
$$


where $\bar{\gamma}>0$ is a known constant. Here, we assume that $\gamma(t)$ is a stationery stochastic process, thus the data dropout rate is independent of the time $t$. In subsequent derivations, we treat $\gamma$ as time invariant.

When the data dropout occurs in multiple communication channels, we can similarly define $\mathcal{E}\left[\gamma_{j}\right]=\bar{\gamma}_{j}>0$ for the $j$ th communication channel. Thus, denote

$$
\Gamma=\operatorname{diag}\left(\gamma_{j}\right)=\left[\begin{array}{cccc}
\gamma_{1} & 0 & \cdots & 0 \\
0 & \gamma_{2} & \cdots & 0 \\
\vdots & \vdots & \ddots & \vdots \\
0 & 0 & \cdots & \gamma_{r}
\end{array}\right]
$$

the corresponding mathematical expectation is

$$
\varepsilon[\Gamma]=\bar{\Gamma},
$$

where $\bar{\Gamma}>0$ is known a priori. iteration is

Due to the data dropout, the plant output received by the controller at the $(i+1)$ th

$$
\Gamma_{i+1} \mathbf{y}_{i+1}(t)
$$

Generally speaking, the occurrences of data dropouts at two iterations are uncorrelated, thus independent. On the other hand, ILC law at the current iteration, the $(i+1)$ th iteration, uses only signals of the previous iteration, namely, ith iteration, as shown in (2.2). Thus $\mathbf{y}_{i+1}(t)$ with the control input $\mathbf{u}_{i+1}(t)$ contains data dropouts upto the $i$ th iteration. Therefore, $\Gamma_{i+1}$ and $\mathbf{y}_{i+1}(t)$ are independent, that is,

$$
\begin{aligned}
\mathcal{E}\left[\Gamma_{i+1} \mathbf{y}_{i+1}(t)\right] & =\mathcal{E}\left[\Gamma_{i+1}\right] \mathcal{\varepsilon}\left[\mathbf{y}_{i+1}(t)\right] \\
& =\bar{\Gamma}_{i+1} \mathcal{\varepsilon}\left[\mathbf{y}_{i+1}(t)\right] .
\end{aligned}
$$

Without the loss of generality, we assume $\varepsilon\left[\Gamma_{i}\right]=\bar{\Gamma}$, namely, the data dropout rate is invariant at different iterations.

Next formulate the one-step communication delay problem. Denote $w(t)$ is a random delay factor with Bernoulli distribution, which takes binary values 0 and 1 that indicate, respectively, the presence and absence of an one-step communication delay. Here we assume that $w(t)$ is a stationery stochastic process, thus the occurrence of the communication delay is independent of the time $t$. In subsequent derivations we treat $w$ as time invariant. With multiple communication channels, we define matrix $W=\operatorname{diag}\left(w_{j}\right)$, where $w_{j}$ denotes the occurrence of communication delay at the $j$ th communication channel. Denote $\varepsilon[w]=\bar{w}$ and $\varepsilon[W]=\bar{W}$. The plant output received by ILC with possible communication delay is formulated by

$$
\mathbf{y}_{i}^{o}(t)=W_{i} \mathbf{y}_{i}(t)+\left[1-W_{i}\right] \mathbf{y}_{i}(t-1)
$$


where $W_{i}$ is the communication delay at the $i$ th iteration. Without the loss of generality, we assume $\varepsilon\left[W_{i}\right]=\bar{W}$, namely, the probability of the communication delay is invariant at different iterations. Analogous to data dropout, assume that communication delay at any two iterations are independent, then $W_{i+1}$ and $W_{i}$ are independent, so are $W_{i+1}$ and $\mathbf{y}_{i+1}(t)$ because $\mathbf{y}_{i+1}(t)$ contains communication delays upto the $i$ th iteration through the ILC law (2.2).

It is worthwhile noting that stochastic variables $\gamma$ and $w$ are not completely independent. A delayed or nondelayed communication occurs only when $\gamma=1$, that is, no data dropout. Hence, we should have the condition probability for data transmission without delay

$$
\operatorname{Prob}[\gamma=1, w=1]=p[\gamma=1] p[w=1 \mid \gamma=1]=\overline{\gamma w}
$$

and the condition probability for data transmission with one-step delay

$$
\begin{aligned}
P[\gamma=1, w=0] & =P[\gamma=1] P[w=0 \mid \gamma=1] \\
& =P[\gamma=1](1-p[w=1 \mid \gamma=1])=\bar{\gamma}(1-\bar{w}) .
\end{aligned}
$$

As a consequence, we have

$$
\varepsilon[\gamma w]=\overline{\gamma w} \text {. }
$$

The relationship between data drop out and communication delay, (2.11), can be extended to multiple channels at the $i$ th iteration

$$
\varepsilon\left[\Gamma_{i} W_{i}\right]=\overline{\Gamma W} .
$$

At the $i$ th iteration, the output signals perturbed by data dropout and one-step communication delay can be expressed as

$$
\tilde{\mathbf{y}}_{i}(t)=\Gamma_{i} \mathbf{y}_{i}^{o}(t)=\Gamma_{i}\left[W_{i} \mathbf{y}_{i}(t)+\left(I-W_{i}\right) \mathbf{y}_{i}(t-1)\right],
$$

where $I$ is a unity matrix of appropriate dimensions. The mathematical expectation of $\tilde{\mathbf{y}}_{i}(t)$ can be derived using the independence property between $\Gamma_{i}, W_{i}$, and $\mathbf{y}_{i}$, as well as the relationship (2.12)

$$
\begin{aligned}
\mathcal{\varepsilon}\left[\tilde{\mathbf{y}}_{i}(t)\right] & =\mathcal{E}\left\{\Gamma_{i}\left[W \mathbf{y}_{i}(t)+(I-W) \mathbf{y}_{i}(t-1)\right]\right\} \\
& =\bar{\Gamma}\left\{\bar{W} \varepsilon\left[\mathbf{y}_{i}(t)\right]+(I-\bar{W}) \mathcal{\varepsilon}\left[\mathbf{y}_{i}(t-1)\right]\right\} .
\end{aligned}
$$

The objective of control design is to seek an appropriate ILC law that can take into consideration data dropout and communication delay concurrently. The following ILC law is adopted

$$
\mathbf{u}_{i+1}(t)=\mathbf{u}_{i}(t)+L \tilde{e}_{i}(t+1)
$$


where

$$
\begin{aligned}
\tilde{\mathbf{e}}_{i}(t+1) & =\Gamma_{i}\left[\mathbf{y}_{d}(t+1)-\tilde{\mathbf{y}}_{i}(t+1)\right] \\
& =\Gamma_{i}\left[\mathbf{y}_{d}(t+1)-W_{i} \mathbf{y}_{i}(t+1)-\left(I-W_{i}\right) \mathbf{y}_{i}(t)\right] \\
& =\Gamma_{i}\left[W_{i} \mathbf{e}_{i}(t+1)+\left(I-W_{i}\right) \mathbf{e}_{i}(t)+\left(I-W_{i}\right) \boldsymbol{\delta}(t)\right],
\end{aligned}
$$

where $\boldsymbol{\delta}(t) \triangleq \mathbf{y}_{d}(t+1)-\mathbf{y}_{d}(t)$.

\section{Convergence Analysis for Left-Invertible Systems $r \geq p$}

In this section, we derive the convergence property of the ILC (2.15) in the presence of data dropout and communication delays.

In ILC, the learning convergence can be derived in terms of either the output tracking error, $\mathbf{e}_{i}(t)$, or the input tracking error, $\Delta \mathbf{u}_{i}(t)$. In this section, we prove the learning convergence property of $\Delta \mathbf{u}_{i}(t)$.

Assumption 3.1. For a given output reference trajectory $\mathbf{y}_{d}(t)$, which is realizable, there exists a unique desired control input $\mathbf{u}_{d}(t) \in \mathbb{R}^{p}$ such that

$$
\begin{gathered}
\mathbf{x}_{d}(t+1)=A \mathbf{x}_{d}(t)+B \mathbf{u}_{d}(t), \\
\mathbf{y}_{d}(t)=C \mathbf{x}_{d}(t),
\end{gathered}
$$

where $\mathbf{u}_{d}(t)$ is uniformly bounded for all $t \in[0, T]$. It is assumed that for all $i \in \mathbb{Z}_{+}, \mathbf{x}_{i}(0)$ is a random variable with $\varepsilon\left[\mathbf{x}_{i}(0)\right]=\mathbf{x}_{0}=\mathbf{x}_{d}(0)$.

Define the input and state errors

$$
\begin{aligned}
& \Delta \mathbf{u}_{i+1} \triangleq \mathbf{u}_{d}(t)-\mathbf{u}_{i+1}(t), \\
& \Delta \mathbf{x}_{i+1} \triangleq \mathbf{x}_{d}(t)-\mathbf{x}_{i+1}(t),
\end{aligned}
$$

then from (2.1) and (3.1), we have

$$
\begin{gathered}
\Delta \mathbf{x}_{i}(t+1)=A \Delta \mathbf{x}_{i}(t)+B \Delta \mathbf{u}_{i}(t) \\
\mathbf{e}_{i}(t)=C \Delta \mathbf{x}_{i}(t)
\end{gathered}
$$

From (2.15), using the relationship (2.12), we have

$$
\begin{aligned}
\mathcal{\varepsilon}\left[\tilde{\mathbf{e}}_{i+1}(t+1)\right] & =\mathcal{E}\left[\Gamma_{i}\left[\mathbf{y}_{d}(t+1)-W_{i} \mathbf{y}_{i}(t+1)-\left(I-W_{i}\right) \mathbf{y}_{i}(t)\right]\right] \\
& =\mathcal{\varepsilon}\left[\Gamma_{i}\left[W_{i} \mathbf{e}_{i}(t+1)+\left(I-W_{i}\right) \mathbf{e}_{i}(t)+\left(I-W_{i}\right)\left(\mathbf{y}_{d}(t+1)-\mathbf{y}_{d}(t)\right)\right]\right] \\
& =\bar{\Gamma}\left\{\bar{W} \mathcal{\varepsilon}\left[\mathbf{e}_{i}(t+1)\right]+(I-\bar{W}) \mathcal{\varepsilon}\left[\mathbf{e}_{i}(t)\right]+(I-\bar{W}) \mathcal{\varepsilon}[\delta(t)]\right\} .
\end{aligned}
$$


Theorem 3.2. Suppose that the update law (2.15) is applied to the networked control system and satisfied the Assumption 3.1. If there exist $\rho$ satisfying

$$
\left\|I_{p}-L \overline{\Gamma W} C B\right\| \leq \rho<1,
$$

then the input error along the iteration axis, $\varepsilon\left[\Delta \mathbf{u}_{i}(t)\right]$, converges to a bound that is proportional to the factor $\boldsymbol{\delta}(t)$.

Proof. First, subtracting $\mathbf{u}_{d}(t)$ from both sides of the ILC law (2.15) yields

$$
\Delta \mathbf{u}_{i+1}(t)=\Delta \mathbf{u}_{i}(t)-L \tilde{\mathbf{e}}_{i}(t+1)
$$

Applying the ensemble operator $\varepsilon[\cdot]$ to both sides of (3.6) and substituting the relationship (3.4) with $\mathbf{e}_{i}(t)=C \Delta \mathbf{x}_{i}(t)$, we obtain

$$
\begin{aligned}
\mathcal{E}\left[\Delta \mathbf{u}_{i+1}(t)\right]= & \mathcal{\varepsilon}\left[\Delta \mathbf{u}_{i}(t)\right]-L \overline{\Gamma W} C \mathcal{E}\left[\Delta \mathbf{x}_{i}(t+1)\right] \\
& -L \bar{\Gamma}(I-\bar{W}) C \mathcal{\varepsilon}\left[\Delta \mathbf{x}_{i}(t)\right]-L \bar{\Gamma}(I-\bar{W}) \boldsymbol{\delta}(t) .
\end{aligned}
$$

Substituting the state error dynamics (3.3) into (3.7) leads to the following relationship:

$$
\begin{aligned}
\mathcal{\varepsilon}\left[\Delta \mathbf{u}_{i+1}(t)\right]= & \left(I_{p}-L \overline{\Gamma W} C B\right) \mathcal{\varepsilon}\left[\Delta \mathbf{u}_{i}(t)\right] \\
& -L[\overline{\Gamma W} C A+\bar{\Gamma}(I-\bar{W}) C] \mathcal{\varepsilon}\left[\Delta \mathbf{x}_{i}(t)\right]-L \bar{\Gamma}(I-\bar{W}) \boldsymbol{\delta}(t) .
\end{aligned}
$$

Define $\rho \triangleq\left\|I_{p}-L \overline{\Gamma W} C B\right\|$.

Now let us handle the second term on the right hand side of (3.8), which is related to $\Delta \mathbf{x}_{i}(t)$. Applying the ensemble operation to the following relationship:

$$
\Delta \mathbf{x}_{i}(t)=A^{t}\left[\mathbf{x}_{d}(0)-\mathbf{x}_{i}(0)\right]+\sum_{k=0}^{t-1} A^{t-1-k} B \Delta \mathbf{u}_{i}(k),
$$

Substituting the relation (3.9) into (3.8), taking the norm $\|\cdot\|_{2}$ on both sides, the following relationship is derived:

$$
\begin{aligned}
\left\|\mathcal{E}\left[\Delta \mathbf{u}_{i+1}(t)\right]\right\|_{2} \leq & \rho\left\|\mathcal{E}\left[\Delta \mathbf{u}_{i}(t)\right]\right\|_{2}+\|L \overline{\Gamma W} C A+L \bar{\Gamma}(I-\bar{W}) C\| a^{t}\left\|\mathbf{x}_{d}(0)-\mathcal{E}\left[\mathbf{x}_{i}(0)\right]\right\|_{2} \\
& +\|L \overline{\Gamma W} C A+L \bar{\Gamma}(I-\bar{W}) C\|\|B\| \sum_{k=0}^{t-1} a^{t-1-k}\left\|\mathcal{E}\left[\Delta \mathbf{u}_{i}(k)\right]\right\|_{2} \\
& +\|L \bar{\Gamma}(I-\bar{W})\|\|\boldsymbol{\delta}(t)\|_{2}
\end{aligned}
$$

where $a \geq\|A\|$ and in this work we choose $a>1$ if $\|A\| \leq 1$. 
In order to handle the exponential term with $a^{t}$ in (3.11), we introduce the $\lambda$ norm. From Assumption 3.1, multiplying both sides of (3.10) by $a^{-\lambda t}$ and taking the supermum over $[0, T]$ yield

$$
\begin{aligned}
\sup _{t \in[0, T]} a^{-\lambda t}\left\|\mathcal{E}\left[\Delta \mathbf{u}_{i+1}(t)\right]\right\|_{2} \leq & \rho \sup _{t \in[0, T]} a^{-\lambda t}\left\|\mathcal{E}\left[\Delta \mathbf{u}_{i}(t)\right]\right\|_{2} \\
& +\beta_{1} \sup _{t \in[0, T]} a^{-\lambda t} \sum_{k=0}^{t-1} a^{t-1-k}\left\|\mathcal{E}\left[\Delta \mathbf{u}_{i}(k)\right]\right\|_{2} \\
& +\beta_{2} \sup _{t \in[0, T]} a^{-\lambda t}\|\boldsymbol{\delta}(t)\|_{2},
\end{aligned}
$$

where $\beta_{1}=\|L \overline{\Gamma W} C A+L \bar{\Gamma}(I-\bar{W}) C\|\|B\|, \beta_{2}=\|L \bar{\Gamma}(I-\bar{W})\|$, and $\eta_{i}=\left\|\mathbf{x}_{d}(0)-\varepsilon\left[\mathbf{x}_{i}(0)\right]\right\|$ that is independent of $t$. Since $\|\boldsymbol{\delta}(t)\|$ is bounded, so is

$$
\mu=\sup _{t \in[0, T]} a^{-\lambda t}\|\boldsymbol{\delta}(t)\|_{2}
$$

Substituting the properties of Lemma A.1 into (3.11) yields

$$
\left\|\mathcal{E}\left[\Delta \mathbf{u}_{i+1}\right]\right\|_{(\lambda, a)} \leq\left(\rho+\beta_{1} \frac{1-a^{-(\lambda-1) T}}{a^{\mathcal{\lambda}}-a}\right)\left\|\mathcal{E}\left[\Delta \mathbf{u}_{i}\right]\right\|_{(\lambda, a)}+\beta_{2} \mu .
$$

Since $0 \leq \rho<1$, it is possible to choose $\lambda$ sufficiently large such that

$$
\rho_{1}=\rho+\beta_{1} \frac{1-a^{-(\lambda-1) T}}{a^{\curlywedge}-a}<1
$$

Therefore we can rewrite (3.13) as

$$
\left\|\mathcal{E}\left[\Delta \mathbf{u}_{i+1}\right]\right\|_{(\lambda, a)} \leq \rho_{1}\left\|\mathcal{E}\left[\Delta \mathbf{u}_{i}\right]\right\|_{(\lambda, a)}+\beta_{2} \mu,
$$

which implies

$$
\lim _{i \rightarrow \infty}\left\|\mathcal{E}\left[\Delta \mathbf{u}_{i}\right]\right\|_{(\lambda, a)} \leq \frac{\beta_{2} \mu}{1-\rho_{1}}
$$

Note that $\mu$ is proportional to $\delta(t)$, namely, the maximum difference between $\mathbf{y}_{d}(t+$ 1) $-\mathbf{y}_{d}(t)$ in $t \in[0, T]$, which is bounded and small when the reference trajectory is smooth or the sampling interval is sufficiently small. When the probability associated with the data communication delay, $\bar{W}$, is known a priori, we can further revise the reference trajectory to an augmented one, such that the resulting $\boldsymbol{\delta}(t)=0$.

Corollary 3.3. Revising the original reference $\mathbf{y}_{d}(t)$ into an augmented one $\mathbf{y}_{d}^{a}(t)=\bar{W} \mathbf{y}_{d}(t)+(I-$ $\bar{W}) \mathbf{y}_{d}(t-1)$, then $\boldsymbol{\delta}(t)=0$ and the ILC (2.15) ensures a zero-tracking error. 
Proof. Note that $\mathcal{\varepsilon}\left[\mathbf{y}_{i}^{o}(t)\right]=\bar{W} \mathcal{\varepsilon}\left[\mathbf{y}_{i}(t)\right]+(I-\bar{W}) \mathcal{E}\left[\mathbf{y}_{i}(t-1)\right]$. Suppose that $\mathbf{y}_{i}(t)=\mathbf{y}_{d}(t)$, then the delay-perturbed output should be $\bar{W} \varepsilon\left[\mathbf{y}_{d}(t)\right]+(I-\bar{W}) \mathcal{\varepsilon}\left[\mathbf{y}_{d}(t-1)\right]$. In other words, the augmented reference trajectory for $\mathbf{y}_{i}^{o}(t+1)$ should be $\mathbf{y}_{d}^{a}(t)=\bar{W} \mathbf{y}_{d}(t)+(I-\bar{W}) \mathbf{y}_{d}(t-1)$. As a result, $\mathbf{y}_{i}^{o}(t)=\mathbf{y}_{d}^{a}(t)$ implies $\mathbf{y}_{i}(t)=\mathbf{y}_{d}(t)$. Now replacing $\mathbf{y}_{d}(t+1)$ in (2.16) with $\mathbf{y}_{d}^{a}(t+1)$, we can derive

$$
\begin{aligned}
\tilde{\mathbf{e}}_{j}(t+1) & =\Gamma_{j}\left[\mathbf{y}_{d}^{a}(t+1)-\mathbf{y}_{j}^{o}(t+1)\right] \\
& =\Gamma_{j}\left[W_{j} \mathbf{y}_{d}(t+1)-W_{j} \mathbf{y}_{j}(t+1)+\left(I-W_{j}\right) \mathbf{y}_{d}(t-1)-\left(I-W_{j}\right) \mathbf{y}_{j}(t)\right] \\
& =\Gamma_{j} W_{j} \mathbf{e}_{j}(t+1)+\Gamma_{j}\left(I-W_{j}\right) \mathbf{e}_{j}(t) .
\end{aligned}
$$

Comparing the above expression with (2.16), we conclude that $\delta(t)=0$, subsequently $\mu=0$, which implies a zero-tracking error according to (3.16).

\section{Convergence Analysis for Right-Invertible Systems $r \leq p$}

In this section, we prove the learning convergence property of $\mathbf{e}_{i}(t)$.

Assumption 4.1. $\left(I_{r}-C B L \overline{\Gamma W}\right)^{-1}$ always exists.

Theorem 4.2. Suppose that the update law (2.15) is applied to the networked control system and satisfied the Assumption 4.1. If

$$
\rho^{\prime} \triangleq\left\|I_{r}-C B L \overline{\Gamma W}\right\|<1
$$

then the tracking error along the iteration axis, $\varepsilon\left[\mathbf{e}_{i}(t)\right]$, converges to a bound that is proportional to the factor $\boldsymbol{\delta}(t)$.

Proof. First note the relationship:

$$
\begin{aligned}
\mathbf{e}_{i+1}(t+1) & =\mathbf{y}_{d}(t+1)-\mathbf{y}_{i+1}(t+1) \\
& =\mathbf{e}_{i}(t+1)+\mathbf{y}_{i}(t+1)-\mathbf{y}_{i+1}(t+1) \\
& =\mathbf{e}_{i}(t+1)+C A\left[\mathbf{x}_{i}(t)-\mathbf{x}_{i+1}(t)\right]+C B\left[\mathbf{u}_{i}(t)-\mathbf{u}_{i+1}(t)\right] \\
\mathbf{x}_{i}(t) & =A^{t} \mathbf{x}_{i}(0)+\sum_{k=0}^{t-1} A^{t-1-k} B \mathbf{u}_{i}(k)
\end{aligned}
$$

Substituting ILC law (2.15), (2.16), and (4.3) into (4.2) yields

$$
\begin{aligned}
\mathbf{e}_{i+1}(t+1)= & \mathbf{e}_{i}(t+1)+C A^{t+1}\left[\mathbf{x}_{i}(0)-\mathbf{x}_{i+1}(0)\right] \\
& -C \sum_{k=0}^{t-1} A^{t-k} B L \widetilde{\mathbf{e}}_{i}(k+1)-C B L \widetilde{\mathbf{e}}_{i}(t+1)
\end{aligned}
$$




$$
\begin{aligned}
= & \mathbf{e}_{i}(t+1)+C A^{t+1}\left[\mathbf{x}_{i}(0)-\mathbf{x}_{i+1}(0)\right] \\
& -C \sum_{k=0}^{t-1} A^{t-k} B L \Gamma_{i}\left[W_{i} \mathbf{e}_{i}(k+1)+\left(I-W_{i}\right) \mathbf{e}_{i}(k)+\left(I-W_{i}\right) \boldsymbol{\delta}(k)\right] \\
& -C B L \Gamma_{i}\left[W_{i} \mathbf{e}_{i}(t+1)+\left(I-W_{i}\right) \mathbf{e}_{i}(t)+\left(I-W_{i}\right) \boldsymbol{\delta}(t)\right] \\
= & \left(I_{r}-C B L \Gamma_{i} W_{i}\right) \mathbf{e}_{i}(t+1)+C A^{t+1}\left[\mathbf{x}_{i}(0)-\mathbf{x}_{i+1}(0)\right] \\
& -C \sum_{k=0}^{t} A^{t-k} B L \Gamma_{i} W_{i} \mathbf{e}_{i}(k+1)-C \sum_{k=0}^{t-1} A^{t-k-1} B L \Gamma_{i}\left(I-W_{i}\right) \mathbf{e}_{i}(k) \\
& -C \sum_{k=0}^{t} A^{t-k} B L \Gamma_{i}\left(I-W_{i}\right) \boldsymbol{\delta}(k) .
\end{aligned}
$$

Assumption 4.3. Assume $\mathcal{\varepsilon}\left[\mathbf{x}_{i+1}(0)\right]-\mathcal{\varepsilon}\left[\mathbf{x}_{i}(0)\right]=0$.

Applying the ensemble operator $\varepsilon[\cdot]$ to both sides of (4.4) and substituting the relationship (4.2), we obtain

$$
\begin{aligned}
\mathcal{\varepsilon}\left[\mathbf{e}_{i+1}(t+1)\right]= & \left(I_{r}-C B L \overline{\Gamma W}\right) \mathcal{\varepsilon}\left[\mathbf{e}_{i}(t+1)\right]-C \sum_{k=0}^{t-1} A^{t-k-1} B L \overline{\Gamma W} \mathcal{\varepsilon}\left[\mathbf{e}_{i}(k+1)\right] \\
& -C \sum_{k=0}^{t} A^{t-k} B L \bar{\Gamma}(I-\bar{W}) \mathcal{\varepsilon}\left[\mathbf{e}_{i}(k)\right]-C \sum_{k=0}^{t} A^{t-k} B L \bar{\Gamma}(I-\bar{W}) \mathcal{\varepsilon}[\boldsymbol{\delta}(t)] .
\end{aligned}
$$

Taking the norm $\|\cdot\|_{2}$ on both sides of (4.5), the following relationship is derived

$$
\begin{aligned}
\left\|\mathcal{E}\left[\mathbf{e}_{i+1}(t+1)\right]\right\|_{2} \leq & \left\|I_{r}-C B L \overline{\Gamma W}\right\|\left\|\mathcal{E}\left[\mathbf{e}_{i}(t+1)\right]\right\|_{2}+\|C\| \sum_{k=0}^{t-1} a^{t-k-1}\|B L\| \overline{\Gamma W}\left\|\mathcal{\varepsilon}\left[\mathbf{e}_{i}(k+1)\right]\right\|_{2} \\
& +\|C\| \sum_{k=0}^{t} a^{t-k}\|B L\| \bar{\Gamma}(I-\bar{W})\left\|\mathcal{\varepsilon}\left[\mathbf{e}_{i}(k)\right]\right\|_{2} \\
& +\|C\| \sum_{k=0}^{t} a^{t-k}\|B L\| \bar{\Gamma}(I-\bar{W})\|\mathcal{\varepsilon}[\boldsymbol{\delta}(t)]\|_{2},
\end{aligned}
$$

where $a \geq\|A\|$ and in this work we choose $a>1$ if $\|A\| \leq 1$. 
In order to handle the exponential term with $a^{t}$ in (4.5), we introduce the $\lambda$ norm. Multiplying both sides of (4.5) by $a^{-\lambda t}$ and taking the supermum over $[0, T]$ yield

$$
\begin{aligned}
\sup _{t \in[0, T]} a^{-\lambda t}\left\|\mathcal{E}\left[\mathbf{e}_{i+1}(t)\right]\right\|_{2} \leq & \sup _{t_{1} \in[0, T]}\|I-C B L \overline{\Gamma W}\| \sup _{t \in[0, T]} a^{-\lambda t}\left\|\mathcal{\varepsilon}\left[\mathbf{e}_{i}(t)\right]\right\|_{2} \\
& +\|C\| \sup _{t \in[0, T]} a^{-\lambda t} \sum_{k=1}^{t} a^{t-k}\|B L\| \overline{\Gamma W}\left\|\mathcal{\varepsilon}\left[\mathbf{e}_{i}(k)\right]\right\|_{2} \\
& +\|C\| \sup _{t \in[0, T]} a^{-\lambda t} \sum_{k=0}^{t-1} a^{t-k-1}\|B L\| \bar{\Gamma}(I-\bar{W})\left\|\mathcal{E}\left[\mathbf{e}_{i}(k)\right]\right\|_{2} \\
& +\|C\| \sup _{t \in[0, T]} a^{-\lambda t} \sum_{k=0}^{t} a^{t-k}\|B L\| \bar{\Gamma}(I-\bar{W})\|\mathcal{\varepsilon}[\boldsymbol{\delta}(t)]\|_{2} .
\end{aligned}
$$

Substituting the properties of Lemma A.2 into (4.7) yields

$$
\left\|\mathcal{E}\left[\mathbf{e}_{i+1}\right]\right\|_{(\lambda, a)} \leq\left(\rho^{\prime}+\beta_{4} \frac{1-a^{-(\lambda-1) T}}{a^{(\lambda-1)}-1}\right)\left\|\mathcal{E}\left[\mathbf{e}_{i}\right]\right\|_{(\lambda, a)}+\beta_{5} \mu,
$$

where $\beta_{3} \triangleq\|C\|\|B L\| \bar{\Gamma}$ and $\beta_{4} \triangleq\|C\|\|B L\| \bar{\Gamma}(I-\bar{W})\left(\left(1-a^{-(\lambda-1) T}\right) /\left(a^{(\lambda-1)}-1\right)\right)$.

Since $0 \leq \rho^{\prime}<1$, it is possible to choose $\lambda$ sufficiently large such that

$$
\rho_{2}=\rho^{\prime}+\beta_{3} \frac{1-a^{-(\lambda-1) T}}{a^{(\lambda-1)}-1}<1 .
$$

Therefore, we can rewrite (4.8) as

$$
\left\|\mathcal{E}\left[\mathbf{e}_{i+1}\right]\right\|_{(\lambda, a)} \leq \rho_{2}\left\|\mathcal{E}\left[\mathbf{e}_{i}\right]\right\|_{(\lambda, a)}+\beta_{4} \mu,
$$

which implies

$$
\lim _{i \rightarrow \infty}\left\|\mathcal{E}\left[\mathbf{e}_{i}\right]\right\|_{(\lambda, a)} \leq \frac{\beta_{4} \mu}{1-\rho_{2}}
$$

\section{Numerical Examples}

Consider the following linear discrete-time system:

$$
\begin{gathered}
\mathbf{x}_{i}(t+1)=\left[\begin{array}{ccc}
0.50 & -0.25 & 1.00 \\
0.15 & 0.30 & -0.50 \\
-0.75 & 0.25 & -0.25
\end{array}\right] \mathbf{x}_{i}(t)+\left[\begin{array}{l}
0 \\
0 \\
1
\end{array}\right] u_{i}(t), \\
y_{i}(t)=\left[\begin{array}{lll}
0 & 0 & 1.0
\end{array}\right] \mathbf{x}_{i}(t),
\end{gathered}
$$


with the initial condition $x_{i}(0)=0$. The desired trajectory is $y_{d}(t)=\sin (2 \pi t / 50)$. The tracking period is $\{0,1, \ldots, 49\}$. The control profile of the first iteration is $u_{0}(t)=0$ for $t=0,1, \ldots, 49$. Two sets of probabilities for the data dropout rate and communication delay are considered, which are $\bar{\gamma}=0.9, \bar{w}=0.9, \bar{\gamma}=0.6$, and $\bar{w}=0.6$, respectively. The learning gain is $L=0.5$, which yields $\|I-L \overline{\gamma w} C B\|=0.595<1$, and $\|I-L \overline{\gamma w} C B\|=0.82<1$ with respect to the two sets of probabilities. The tracking performance of two ILC algorithms is given in Figure 2, where Max Error denotes the maximum absolute error of each iteration.

\section{Conclusion}

In this work, we address a class of networked control system problems with random data dropout and communication delay. D-type ILC is applied to handle this remote control systems problem with repeated tracking tasks. Through analysis, we illustrate the desired convergence property of the ILC. Although we focus on one-step communication delay in this work, the results could be extended to multiple delays, which is one of our ongoing research topics. In our future work, we will also explore the extension to more generic nonlinear dynamic processes.

\section{Appendix}

Lemma A.1. For all $a>1$, for all $\lambda>1$, for all $i \in \mathbb{Z}_{+}$, the inequality:

$$
\sup _{t \in[0, T]} a^{-\lambda t} \sum_{\tau=0}^{t-1} a^{t-1-\tau}\left\|\mathcal{E}\left[\Delta \mathbf{u}_{i}(\tau)\right]\right\|_{2} \leq \frac{1-a^{-(\lambda-1) T}}{a^{\lambda}-a}\left\|\mathcal{E}\left[\Delta \mathbf{u}_{i}\right]\right\|_{(\lambda, a)}
$$

holds.

Proof. Consequently

$$
\begin{aligned}
\sup _{t \in[0, T]} a^{-\lambda t} \sum_{\tau=0}^{t-1} a^{t-1-\tau}\left\|\mathcal{E}\left[\Delta \mathbf{u}_{i}(\tau)\right]\right\|_{2} & =a^{-1} \sup _{t \in[0, T]} a^{-t(\lambda-1)} \sum_{\tau=0}^{t-1} a^{-\lambda \tau}\left\|\mathcal{E}\left[\Delta \mathbf{u}_{i}(\tau)\right]\right\| a^{(\lambda-1) \tau} \\
& \leq a^{-1}\left\|\mathcal{E}\left[\Delta \mathbf{u}_{i}\right]\right\|_{(\lambda, a)} \sup _{t \in[0, T]} a^{-t(\lambda-1)} \frac{a^{(\lambda-1) t}-1}{a^{\lambda-1}-1} \\
& \leq\left(\frac{1-a^{-(\lambda-1) T}}{a^{\lambda}-a}\right)\left\|\mathcal{E}\left[\Delta \mathbf{u}_{i}\right]\right\|_{(\lambda, a)} .
\end{aligned}
$$

Lemma A.2. For all $a>1$, for all $\lambda>1$, for all $i \in \mathbb{Z}_{+}$, the inequalities

$$
\begin{aligned}
& \sup _{t \in[0, T]} a^{-\lambda t} \sum_{k=0}^{t-1} a^{t-1-k}\left\|\mathcal{E}\left[\mathbf{e}_{i}(k)\right]\right\|_{2} \leq \frac{1-a^{-(\lambda-1) T}}{a^{(\lambda-1)}-1}\left\|\mathcal{E}\left[\mathbf{e}_{i}\right]\right\|_{(\lambda, a)}, \\
& \sup _{t \in[0, T]} a^{-\lambda t} \sum_{k=1}^{t} a^{t-1-k}\left\|\mathcal{E}\left[\mathbf{e}_{i}(k)\right]\right\|_{2} \leq \frac{1-a^{-(\lambda-1) T}}{a^{(\lambda-1)}-1}\left\|\mathcal{E}\left[\mathbf{e}_{i}\right]\right\|_{(\lambda, a)}
\end{aligned}
$$

hold. 


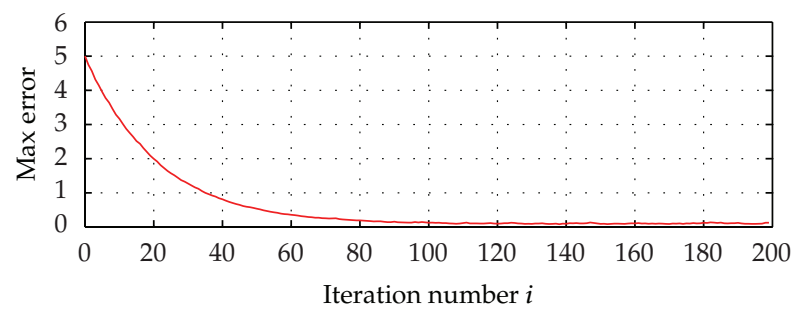

(a)

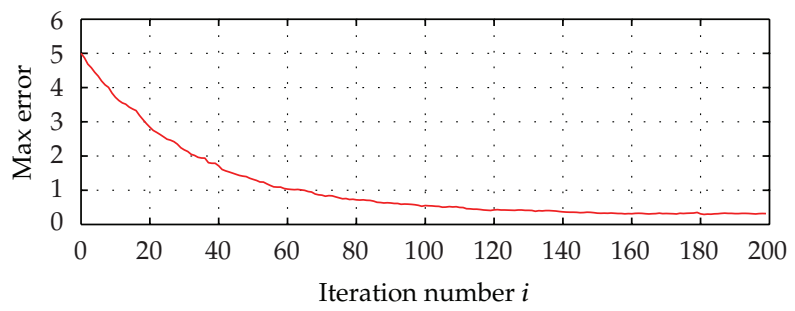

(b)

Figure 2: The tracking error profiles for the discrete-time linear system with data dropout and one-step communication delay. (a) is learning results with the data dropout rate $\bar{\gamma}=0.9$ and communication delay rate $\bar{w}=0.9$. (b) is learning results with the data dropout rate $\bar{\gamma}=0.6$ and communication delay rate $\bar{w}=0.6$.

Proof. Consequently

$$
\begin{aligned}
\sup _{t \in[0, T]} a^{-\lambda t} \sum_{k=0}^{t-1} a^{t-1-k}\left\|\mathcal{E}\left[\mathbf{e}_{i}(k)\right]\right\|_{2} & \leq \sup _{t \in[0, T]} a^{-\lambda t} \sum_{k=0}^{t} a^{t-k}\left\|\mathcal{E}\left[\mathbf{e}_{i}(k)\right]\right\|_{2} \\
& =\sup _{t \in[0, T]} a^{-t(\lambda-1)} \sum_{k=0}^{t} a^{-\lambda k}\left\|\mathcal{E}\left[\mathbf{e}_{i}(k)\right]\right\| a^{(\lambda-1) k} \\
& \leq\left\|\mathcal{E}\left[\mathbf{e}_{i}\right]\right\|_{(\lambda, a)} \sup _{t \in[0, T]} a^{-t(\lambda-1)} \frac{a^{(\lambda-1) t}-1}{a^{\lambda-1}-1} \\
& \leq\left(\frac{1-a^{-(\lambda-1) T}}{a^{\lambda-1}-1}\right)\left\|\mathcal{E}\left[\mathbf{e}_{i}\right]\right\|_{(\lambda, a)}, \\
\sup _{t \in[0, T]} a^{-\lambda t} \sum_{k=1}^{t} a^{t-1-k}\left\|\mathcal{E}\left[\mathbf{e}_{i}(k)\right]\right\|_{2} & \leq \sup _{t \in[0, T]} a^{-\lambda t} \sum_{k=0}^{t} a^{t-k}\left\|\mathcal{E}\left[\mathbf{e}_{i}(k)\right]\right\|_{2} \\
& =\sup _{t \in[0, T]} a^{-t(\lambda-1)} \sum_{k=0}^{t} a^{-\lambda k}\left\|\mathcal{E}\left[\mathbf{e}_{i}(k)\right]\right\| a^{(\lambda-1) k} \\
& \leq\left\|\mathcal{E}\left[\mathbf{e}_{i}\right]\right\|_{(\lambda, a)} \sup _{t \in[0, T]} a^{-t(\lambda-1)} \frac{a^{(\lambda-1) t}-1}{a^{\lambda-1}-1} \\
& \leq\left(\frac{1-a^{-(\lambda-1) T}}{a^{\lambda-1}-1}\right)\left\|\mathcal{E}\left[\mathbf{e}_{i}\right]\right\|_{(\lambda, a)} .
\end{aligned}
$$




\section{Acknowledgments}

This work is supported by the National Natural Science Foundation of China (Grants no. 60736021 and no. 60721062), The 973 Program of China (Grant no. 2009CB320603).

\section{References}

[1] Y. Q. Chen and K. L. Moore, "Harnessing the nonrepetitiveness in iterative learning control," in Proceedings of the 41st IEEE Conference on Decision and Control, vol. 3, pp. 3350-3355, Las Vegas, Nev, USA, 2002.

[2] D. A. Bristow, M. Tharayil, and A. G. Alleyne, "A survey of iterative learning control," IEEE Control Systems Magazine, vol. 26, no. 3, pp. 96-114, 2006.

[3] Z. Bien and I. M. Huh, "Higher-order iterative learning control algorithm," IEE Proceedings D, vol. 136, no. 3, pp. 105-112, 1989.

[4] J.-X. Xu and Y. Tan, Linear and Nonlinear Iterative Learning Control, Springer, Berlin, Germany, 2003.

[5] C.-J. Chien, "A discrete iterative learning control for a class of nonlinear time-varying systems," IEEE Transactions on Automatic Control, vol. 43, no. 5, pp. 748-752, 1998.

[6] M. X. Sun and D. Wang, "Iterative learning control with initial rectifying action," Automatica, vol. 38, no. 8, pp. 1177-1182, 2002.

[7] K.-H. Park, "An average operator-based PD-type iterative learning control for variable initial state error," IEEE Transactions on Automatic Control, vol. 50, no. 6, pp. 865-869, 2005.

[8] S. S. Saab, "A discrete-time stochastic learning control algorithm," IEEE Transactions on Automatic Control, vol. 46, no. 6, pp. 877-887, 2001.

[9] Y. Q. Chen, Z. Gong, and C. Y. Wen, "Analysis of a high-order iterative learning control algorithm for uncertain nonlinear systems with state delays," Automatica, vol. 34, no. 3, pp. 345-353, 1998.

[10] H.-S. Ahn, K. L. Moore, and Y. Q. Chen, "Discrete-time intermittent iterative learning controller with independent data dropouts," in Proceedings of the 17th World Congress (IFAC '08), Seoul, Korea, 2008.

[11] J. Lam, H. Gao, and C. Wang, "Stability analysis for continuous systems with two additive timevarying delay components," Systems E Control Letters, vol. 56, no. 1, pp. 16-24, 2007.

[12] B. Tang, G. P. Liu, and W. H. Gui, "Improvement of state feedback controller design for networked control systems," IEEE Transactions on Circuits and Systems, vol. 55, no. 5, pp. 464-468, 2008.

[13] T. C. Yang, "Networked control system: a brief survey," IEE Proceedings Control Theory and Applications, vol. 153, no. 4, pp. 403-412, 2006.

[14] J. Hespanha, "Stochastic hybrid systems: application to communication networks," in Lecture Notes in Computer Science, vol. 2993, pp. 387-401, Springer, New York, NY, USA, 2004.

[15] H. Gao and C. Wang, "Delay-dependent robust $H_{\infty}$ and $L_{2}-L_{\infty}$ filtering for a class of uncertain nonlinear time-delay systems," IEEE Transactions on Automatic Control, vol. 48, no. 9, pp. 1661-1666, 2004.

[16] H. Gao and C. Wang, "A delay-dependent approach to robust $H_{\infty}$ filtering for uncertain discrete-time state-delayed systems," IEEE Transactions on Signal Processing, vol. 52, no. 6, pp. 1631-1640, 2004.

[17] F. W. Yang, Z. D. Wang, Y. S. Hung, and M. Gani, " $H_{\infty}$ control for networked systems with random communication delays," IEEE Transactions on Automatic Control, vol. 51, no. 3, pp. 511-518, 2006.

[18] X. H. Bu and Z. S. Hou, "Stability of iterative learning control with data dropout via asynchonous dynamical system," International Journal of Automation and Computing, vol. 8, no. 1, pp. 29-36, 2011.

[19] L. Zhou, Z. J. Zhang, G. P. Lu, and X. Q. Xiao, "Stabilization of discrete-time networked control systems with nonlinear perturbation," in Proceedings of the 27th Chinese Control Conference (CCC '08), pp. 266-270, 2008.

[20] B. G. Marieke, M. Cloosterman, N. van de Wouw, W. P. M. H. Heemels, and H. Nijmeijer, "Stability of networked control systems with uncertain time-varying delays," IEEE Transactions on Automatic Control, vol. 54, no. 7, pp. 1575-1580, 2009.

[21] S. Hu and W. Y. Yan, "Stability robustness of networked control systems with respect to packet loss," Automatica, vol. 43, no. 7, pp. 1243-1248, 2007. 


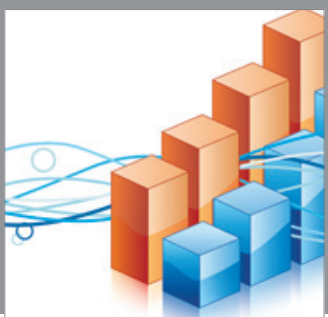

Advances in

Operations Research

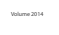

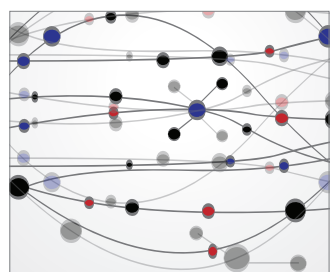

\section{The Scientific} World Journal
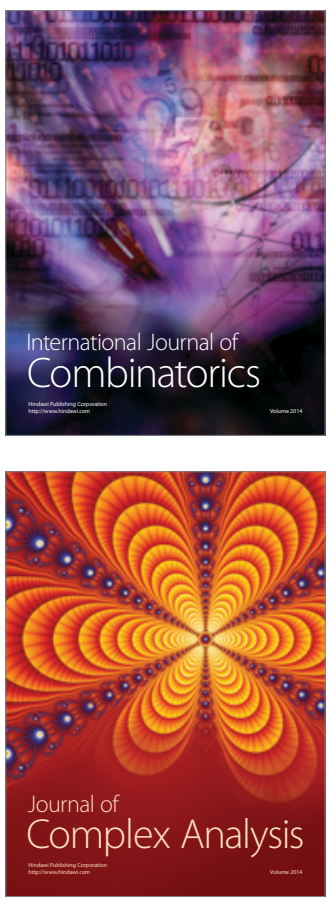

International Journal of

Mathematics and

Mathematical

Sciences
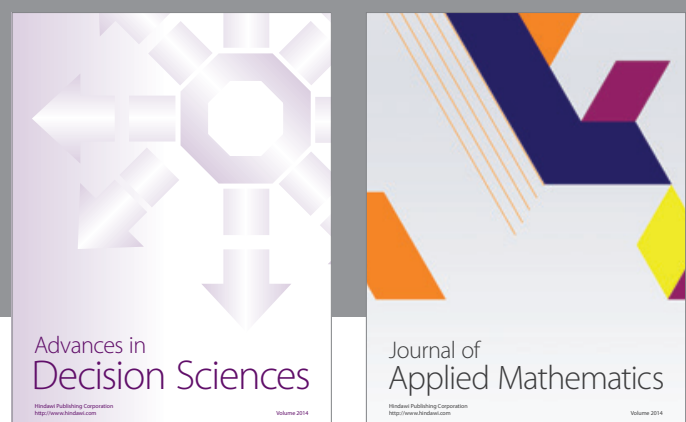

Journal of

Applied Mathematics
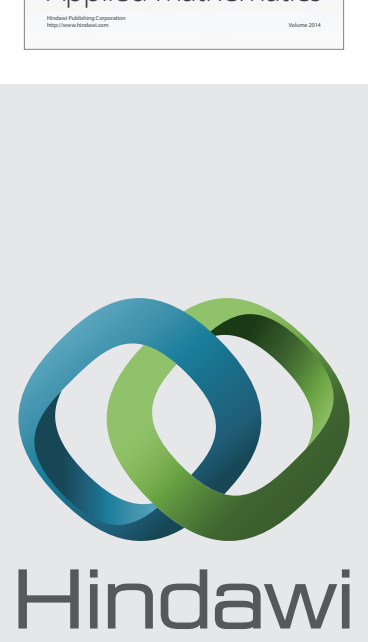

Submit your manuscripts at http://www.hindawi.com
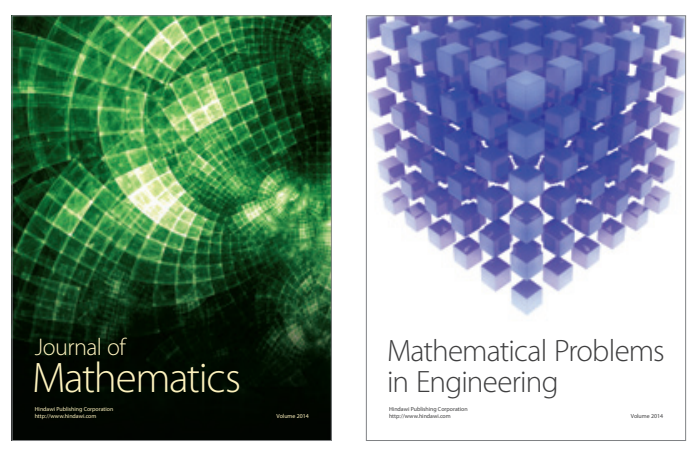

Mathematical Problems in Engineering
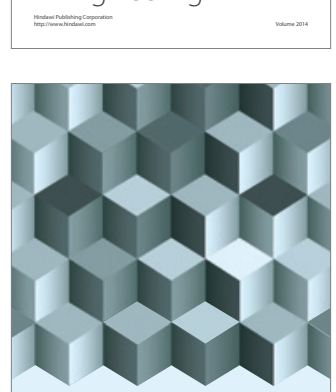

Journal of

Function Spaces
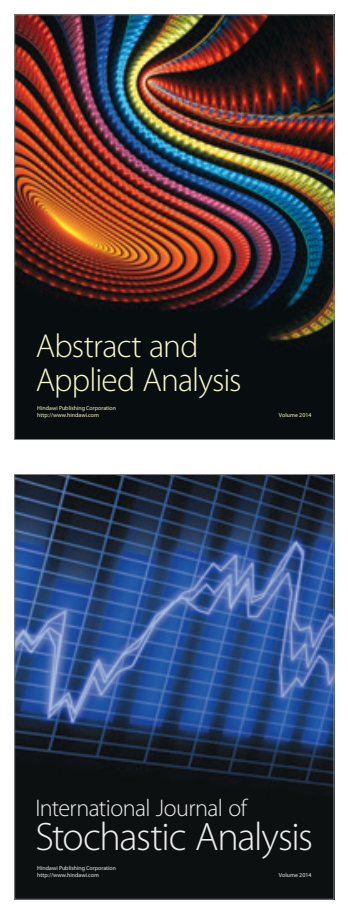

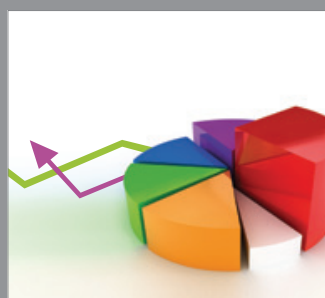

ournal of

Probability and Statistics

Promensencen
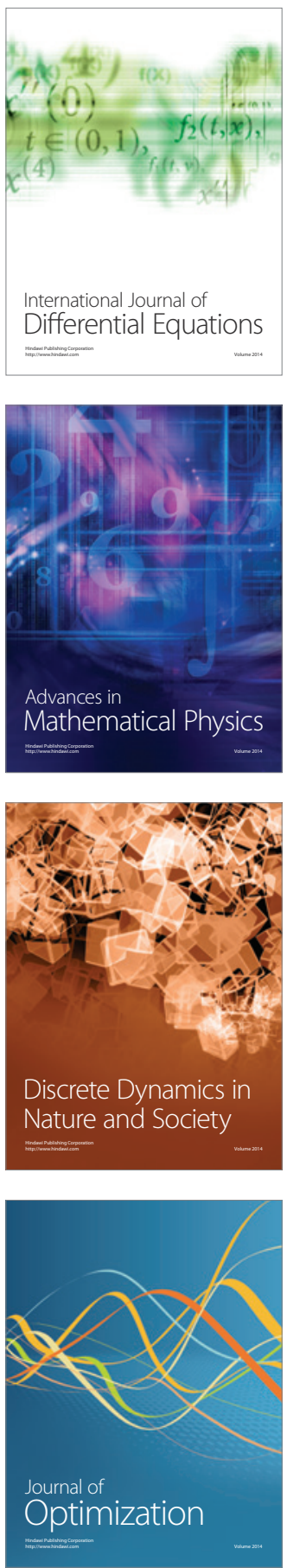